\title{
Atuação da enfermagem na promoção à segurança do paciente
}

\author{
Bruna Oliveira dos Santos*, Adriana de Paula Congro Michelone, M.Sc.**
}

\begin{abstract}
${ }^{*}$ Graduanda do Curso de Enfermagem da Faculdade de Medicina de Marília, ${ }^{* *}$ Docente da disciplina Enfermagem Clínica da Faculdade de Medicina de Marilia
\end{abstract}

\begin{abstract}
Resumo
Introdução: A segurança do paciente vem sendo discutida pela Enfermagem devido ao aumento de problemas relacionados a este tema e porque a equipe de enfermagem exerce papel fundamental nos processos que envolvem a atenção ao paciente. Objetivo: Analisar publicaçóes sobre a ocorrência de eventos adversos em serviços de saúde, identificando as medidas preventivas utilizadas pela enfermagem para promover a segurança dos pacientes hospitalizados. Material e métodos: Pesquisa bibliográfica, feita por busca eletrônica, resultando em 14 artigos. Resultados: Apesar de a segurança do paciente ser importante, isso ainda é pouco estudado. O centro cirúrgico é reconhecido como um dos locais mais favoráveis à ocorrência de eventos adversos. Uma das estratégias para prevenção é o gerenciamento de risco. A equipe de enfermagem precisa estar preparada e qualificada para solucionar intercorrências, sendo essencial a identificação e a notificação dos casos para garantir a minimização dos erros. Conclusão: As instituiçóes de saúde devem enfatizar a divulgação dos conceitos sobre os eventos adversos para conseguir realizar atendimentos mais eficazes e seguros.
\end{abstract}

Palavras-chave: segurança do paciente, gerenciamento de segurança, equipe de enfermagem, cuidados de enfermagem.

\begin{abstract}
Nursing actions on promoting patient safety

Introduction: The patient safety is a subject that has been discussed in the Nursing due to increase of problems related to this theme, and also because the nursing team plays an important role in the patients care process. Objective: To analyze studies concerned about incidence of adverse events in health services, identifying nursing preventive measures used to promote patient safety in hospital. Methods: Literature review, which used electronic research, resulting in 14 articles. Results: Although patient safety is important, there are still few studies on this matter. The surgery center is recognized as the place that favors the occurrence of adverse events. One strategy for prevention is safety management. The Nursing team should be prepared and qualified for solving complications during the procedures, and to make sure that they are identified and notified in order to minimize mistakes. Conclusion: The health institutes should emphasize the need to make public the concepts about adverse events, in order to carry out effective and safety treatment.
\end{abstract}

Key-words: patient safety, safety management, nursing team, nursing care. 


\section{Resumen}

\section{Actuación de enfermería en la promoción de seguridad del paciente}

Introducción: La seguridad del paciente es un tema que ha sido discutido por la Enfermería debido al aumento de los problemas relacionados con este tema, y, porque el equipo de enfermería desempeńa un papel fundamental en los procesos relacionados con la atención al paciente. Objetivo: Analizar los estudios sobre la ocurrencia de eventos adversos en los servicios de salud, mediante la identificación de las medidas preventivas adoptadas por la enfermería para promover la seguridad de los pacientes hospitalizados. Material y métodos: Investigación literaria realizada a través de búsqueda electrónica, que resultó en 14 artículos. Resultados: Aunque la seguridad del paciente es importante, todavía hay pocos estudios sobre el tema. El centro de cirugía es conocido como uno de los lugares más favorables para la ocurrencia de eventos adversos. Una estrategia para la prevención es la gestión de riesgos. El equipo de enfermería necesita estar capacitado y calificado para resolver problemas que pueden surgir, y es esencial identificar y notificar los casos para asegurar la minimización de errores. Conclusión: Las instituciones de salud deben promover la divulgación de conceptos de eventos adversos, para lograr una atención más efectiva y segura.

Palabras-clave: seguridad del paciente, administración de la seguridad, grupo de enfermería, atención de enfermería.

\section{Introdução}

A segurança do paciente é assunto que vem sendo discutido pela Enfermagem nos últimos anos. Devido ao aumento de problemas relacionados a este tema na área da saúde, tornou-se um grave problema de saúde pública. A segurança do paciente é reforçada pela "redução e/ ou atenuação de atos considerados inseguros, atrelados ao sistema de assistência à saúde, bem como ao emprego das melhores práticas, no intuito de obter os resultados esperados" [1].

Assim, foi desenvolvida pelo Conselho Regional de Enfermagem de Sáo Paulo, no ano de 2010, a cartilha "10 passos para a segurança do paciente", que contempla os principais pontos que teriam impacto direto na prática assistencial de enfermagem, podendo ser implementados em diversos ambientes de cuidados. Então, a partir daí, esse Conselho assumiu o compromisso de promover uma grande campanha pela segurança do paciente, esclarecendo a categoria de enfermagem e chamando-a a responsabilidade de lançar um novo olhar sobre suas práticas cotidianas e identificar falhas possíveis de gerar erros no processo [2].

Esta campanha tendo sido muito importante para mostrar aos profissionais que eventos adversos (EAs) podem ser evitados. Carneiro et al. [3] conceituam eventos adversos como incidentes indesejáveis, porém passíveis de prevenção e que ocorrem durante a prestaçáo do cuidado à saúde, resultando em danos ao paciente, gerando comprometimento da estru- tura ou função do corpo e/ou algum efeito nocivo, como doença, lesão, incapacidade, ou morte. Esses eventos adversos podem ser de caráter físico, social e/ou psicológico.

Esses EAs são responsáveis pelo aumento do tempo de permanência do paciente nos hospitais, $o$ que eleva os custos para o sistema de saúde. A partir disso, fica evidente a importância de as instituiçōes reconhecerem a fragilidade e a vulnerabilidade de seus sistemas para que possam identificar as origens dos erros de forma a corrigi-los, tornando a assistência ao paciente mais eficaz e segura [1].

Apesar do grande número de eventos adversos, ainda há baixa qualidade e quantidade de seus registros, pois são vistos como erros individuais de profissionais de saúde e não como deficiências no sistema, punindo-se apenas os profissionais envolvidos. A instituição de saúde deve estimular a cultura da não punição e, consequentemente, incentivar a notificação dos eventos adversos, utilizando-a para implementar açóes que previnam sua ocorrência [4].

Vale destacar que a Organização Mundial da Saúde (OMS) lançou, em 2008, o protocolo "Cirurgias seguras salvam vidas", com objetivo de discutir as açóes preconizadas pela OMS para a segurança do paciente frente ao procedimento cirúrgico [1].

No Brasil, também foi divulgado um manual mostrando medidas para melhorar a segurança do paciente cirúrgico, reconhecendo que, apesar da enorme melhoria no conhecimento sobre segurança cirúrgica, pelo menos metade dos EAs ocorre durante a assistência cirúrgica [5]. 
É importante lembrar que a enfermagem tem papel fundamental no que se refere à segurança do paciente, pois permanece na assistência em tempo integral. Assim, é dela a responsabilidade de realizar os cuidados certos, no momento certo, da maneira certa, para a pessoa certa, objetivando alcançar os melhores resultados possíveis, por serem esses os princípios que fundamentam a qualidade da assistência. Então os enfermeiros, quando capacitados, podem prevenir erros ou detectar precocemente qualquer tipo de evento adverso [6].

Como a segurança é um dos critérios básicos para garantir a qualidade da assistência ao paciente, a adoção de estratégias para redução de erros e eventos adversos em instituiçōes de saúde é fundamental [7].

Desta forma, a presente pesquisa tem como objetivo analisar os estudos publicados sobre a ocorrência de eventos adversos em serviços de saúde, identificando as medidas preventivas utilizadas pela enfermagem para promover a segurança dos pacientes hospitalizados.

\section{Material e métodos}

Trata-se de uma pesquisa bibliográfica, realizada por meio de busca na base de dados Lilacs e BEDENF. Foram utilizados os seguintes descritores: (eventos and adversos) or (segurança and pacientes) palavras do título or (gerencias and riscos) palavras do resumo.

Para a seleção dos artigos foram definidos alguns critérios: artigos publicados em português; artigos na íntegra que retratassem a temática referente à segurança do paciente submetido à cirurgia; e artigos publicados e indexados no período de 2002 a 2012.

Foram encontrados 323 estudos, sendo 91 artigos da BEDENF e 232 da Lilacs. Após a leitura dos títulos e dos resumos, selecionaram-se 14 artigos, tendo sido descartados aqueles que não respondiam à questão norteadora, além de teses, dissertaçóes, capítulos de livros e os repetidos.

Os artigos selecionados foram lidos, na íntegra, e analisados, visando constatar se respondiam à questâo de investigaçáo para a elaboração desta pesquisa: que açóes de enfermagem devem ser realizadas para garantir a segurança dos pacientes hospitalizados, em especial daqueles submetidos à cirurgia?

\section{Resultados}

Nota-se que, apesar de a busca ter sido feita no período entre 2002 e 2012, foram encontrados estudos a partir de 2004. No ano de 2010, houve maior número de publicaçóes, seguido pelos anos de 2011 e 2012. Portanto, pode-se afirmar que a maioria dos estudos é recente.

O periódico que teve maior número de estudos publicados foi a Revista de Enfermagem da UERJ, com o total de três. Já os periódicos da Revista Brasileira de Epidemiologia, da Revista da SOBECC e da Revista da Escola de Enfermagem da USP apresentaram duas publicaçóes cada uma. Os outros cincos periódicos tiveram apenas uma publicação, totalizando nove revistas.

\section{Discussão}

A segurança depende das pessoas, de sua forma de agir e reagir, de hábitos seguros e de processos de trabalho bem organizados, principalmente na área da saúde, uma vez que qualquer equívoco pode causar danos severos e até irreversíveis a outras pessoas [8].

Uma das estratégias utilizadas é o gerenciamento de risco, que determina a aplicação de processos sistemáticos, visando promover a avaliação e o controle de riscos, bem como a prevenção de eventos adversos que afetam a saúde dos indivíduos [9].

Vale ressaltar que o Centro Cirúrgico (CC) é reconhecido como um dos locais mais complexos do hospital e o mais favorável para a ocorrência de eventos adversos por causa das próprias características do cuidar, da diversidade dos procedimentos cirúrgicos e dos diagnósticos, bem como $\mathrm{da}$ intensa circulação de vários profissionais. E, apesar de esses procedimentos terem a intençáo de salvar vidas, estatísticas apontam que a falha de segurança nos processos de assistência cirúrgica pode causar danos [10]. Corroborando essa idéia, um estudo também mostra que o CC é o local onde mais acontecem eventos adversos: dos 1.103 pacientes avaliados, 38 tiveram eventos adversos e $78,1 \%$ desses foram cirúrgicos. Observou-se também que a taxa de mortalidade dos pacientes com EAs cirúrgicos foi de 18,4\% [11]. Nota-se que dentre os artigos examinados, nove trazem o conceito de eventos adversos associados à segurança do paciente. Os EAs são como injúrias não intencionais consequentes da atenção à saúde, não relacionadas à evolução natural da doença de base, que acarretam em lesóes mensuráveis nos pacientes afetados e/ou prolongamento do tempo de internação e/ou óbito [12]. 
Quadro 1 - Artigos analisados na base de dados Lilacs e BEDENF sobre segurança do paciente.

\begin{tabular}{|c|c|c|c|}
\hline & Título do artigo & Autores & Periódico \\
\hline 1 & $\begin{array}{l}\text { Avaliação de eventos adversos cirúrgicos em hospitais do } \\
\text { Rio de Janeiro. }\end{array}$ & Moura MLO, Mendes W. & $\begin{array}{l}\text { Rev Bras Epidemiol } \\
2012 ; 15(3): 523-35\end{array}$ \\
\hline 2 & $\begin{array}{l}\text { Clima de segurança do paciente: percepção dos profis- } \\
\text { sionais de enfermagem. }\end{array}$ & Rigobello MCG, et al. & $\begin{array}{l}\text { Acta Paul Enferm } \\
2012 ; 25(5): 728-35\end{array}$ \\
\hline 3 & $\begin{array}{l}\text { Eventos adversos: instrumento de avaliação do desempe- } \\
\text { nho em centro cirúrgico de um hospital universitário. }\end{array}$ & Souza LP, et al. & $\begin{array}{l}\text { Rev Enferm UERJ } \\
2011 ; 19(1): 127-33\end{array}$ \\
\hline 4 & $\begin{array}{l}\text { O estresse de acadêmicas de enfermagem e a segurança } \\
\text { do paciente. }\end{array}$ & Santos VPE, Raünz V. & $\begin{array}{l}\text { Rev Enferm UERJ } \\
2011 ; 19(4): 616-20 .\end{array}$ \\
\hline 5 & $\begin{array}{l}\text { Evento adverso em terapia intensiva: o que sabem os } \\
\text { profissionais de enfermagem. }\end{array}$ & $\begin{array}{l}\text { Silva RCL, Cunha JJSA, } \\
\text { Moreira CLS. }\end{array}$ & $\begin{array}{l}\text { Rev Pesqui Cuid } \\
\text { Fundam } 2011 ; 3(2) \text { : } \\
1848-55\end{array}$ \\
\hline 6 & $\begin{array}{l}\text { Eventos adversos na clínica cirúrgica de um hospital uni- } \\
\text { versitário: instrumento de avaliação da qualidade. }\end{array}$ & Carneiro FS, et al. & $\begin{array}{l}\text { Rev Enferm UERJ } \\
2011 ; 19(2): 204-11 .\end{array}$ \\
\hline 7 & $\begin{array}{l}\text { O erro médico e a violação às normas e prescrições em } \\
\text { saúde: uma discussão teórica na área de segurança do } \\
\text { paciente. }\end{array}$ & $\begin{array}{l}\text { Nascimento, NB, Travassos } \\
\text { CMR. }\end{array}$ & $\begin{array}{l}\text { Physis (Rio J.) } \\
2010 ; 20(2): 625-51 .\end{array}$ \\
\hline 8 & $\begin{array}{l}\text { Gerenciamento de riscos: qualidade na assistência perio- } \\
\text { peratória. }\end{array}$ & $\begin{array}{l}\text { Oliveira BC, Caregnato RC, } \\
\text { Hoefel HHK. }\end{array}$ & $\begin{array}{l}\text { Rev SOBECC } \\
2010 ; 15(4): 21-27 \\
\end{array}$ \\
\hline 9 & $\begin{array}{l}\text { Segurança do paciente em cirurgia oncológica: experiên- } \\
\text { cia do Instituto do Câncer do Estado de São Paulo. }\end{array}$ & Vendramini RCR, et al. & $\begin{array}{l}\text { Rev Esc Enferm USP } \\
2010 ; 44(3): 827-32\end{array}$ \\
\hline 10 & $\begin{array}{l}\text { Eventos adversos: análise de um instrumento de notifica- } \\
\text { ção utilizado no gerenciamento de enfermagem. }\end{array}$ & $\begin{array}{l}\text { Paiva MCMS, Paiva SAR, } \\
\text { Berti HW. }\end{array}$ & $\begin{array}{l}\text { Rev Esc Enferm USP } \\
2010 ; 44(2): 287-94\end{array}$ \\
\hline 11 & $\begin{array}{l}\text { Fortalezas e ameaças em torno da segurança do paciente } \\
\text { segundo a opinião dos profissionais de enfermagem. }\end{array}$ & $\begin{array}{l}\text { Ques AAM, Montoro CH, } \\
\text { González MG. }\end{array}$ & $\begin{array}{l}\text { Rev Latinoam Enferm } \\
2010 ; 18(3): 42-49\end{array}$ \\
\hline 12 & $\begin{array}{l}\text { Estratégias de prevenção de eventos adversos na sala } \\
\text { operatória. }\end{array}$ & $\begin{array}{l}\text { Cunha ALM, Acunã AA, } \\
\text { Bispo DM. }\end{array}$ & $\begin{array}{l}\text { Rev SOBECC } \\
2007 ; 12(3): 20-23\end{array}$ \\
\hline 13 & $\begin{array}{l}\text { Revisão dos estudos de avaliação da ocorrência de even- } \\
\text { tos adversos em hospitais. }\end{array}$ & MendesW, et al. & $\begin{array}{l}\text { Rev Bras Epidemiol } \\
2005 ; 8(4): 393-406\end{array}$ \\
\hline 14 & Eventos adversos - O que são? & Gallotti RMD. & $\begin{array}{l}\text { Rev Assoc Med Bras } \\
2004 ; 50(2): 114\end{array}$ \\
\hline
\end{tabular}

O evento adverso é considerado um problema de saúde pública, sendo relacionado à administração de medicamentos, de sangue e hemoderivados ou ao uso de artigos e de equipamentos médico-hospitalares. Esse problema precisa ser tratado com maior atenção e frequência como objeto de investigação. O resultado das pesquisas poderá trazer importantes contribuiçóes para definição de estratégias que possam garantir maior segurança ao paciente, pela reduçáo da ocorrência de EAs relacionados à assistência a saúde [4].

Nesse contexto é importante a avaliação dos indicadores de resultados. Trata-se de um instrumento gerencial capaz de representar quali ou quantitativamente a assistência. Sem eles é impossível uma avalição objetiva de qualidade [3].

A frequência de erros depende dos critérios utilizados, variando de 2,9 a $39 \%$ das admissóes, sendo que 18 a $83 \%$ poderiam ser evitados. Porém, a frequência dos diferentes tipos de erros é variável entre as diversas instituiçóes de saúde. Dos erros analisados no estudo, 27,6\% eram devidos à negligência do profissional, $70,5 \%$ resultou em incapacidade em tempo inferior a seis meses, e 13,6\% em mortes [7].

Em comparação com o de outros países, o percentual dos Estados Unidos e do Canadá revela que os procedimentos cirúrgicos foram os que mais apresentaram erros. No Canadá foram identificados $51,4 \%$ de erros em um total de 1.133 EAs; já nos Estados Unidos a porcentagem foi de 44,9\% [7].

$\mathrm{Na}$ Califórnia, a incidência de EAs, em 1874, foi de 4,6 por 100 pacientes. Dez anos depois, a incidência em Nova York foi de 3,7 por 100 pacientes, sendo que um quarto desses foi devido à negligência. Já na Nova Zelândia, no ano de 1998, foi analisada uma amostra de 6.579 prontuários, 
na qual se notou a incidência de $11,3 \%$ de EAs. $\mathrm{Na}$ Dinamarca, o índice foi de $9 \%$ de erros em uma amostra de 1.097 prontuários. Na Austrália, em 1992, a incidência de EAs foi de 16,5 por 100 pacientes, considerando uma amostra de 14.479 prontuários. Este país foi o que teve o maior índice dentre os países analisados [13].

Os Estados Unidos foram também citados em outros estudos [10]. No país, por ano, acontecem um milhão de EAs evitáveis e 100 mil pessoas morrem vítimas desses eventos. No mesmo estudo, os autores relatam que no Brasil a incidência de EAs também é alta e corresponde a de 38,4\%. Para cada 100 pacientes internados, oito sofrem um ou mais EAs e, no centro cirúrgico, 14\% dos erros ocorrem por negligência.

Quanto à natureza dos EAs na clínica cirúrgica, $61,36 \%$ estáo relacionados com a retirada de sondas, drenos e cateteres, além da queda dos pacientes com $18,56 \%$. Há também os procedimentos alérgicos e evasão com 5,3\% cada, úlceras por pressão $(4,92 \%)$, erros de medicação $(2,65 \%)$, procedimentos médi$\cos (1,14 \%)$, e hemoderivados e queimaduras com $0,38 \%$ cada [3].

Ao ser submetido a um procedimento cirúrgico, o paciente está exposto a muitos riscos, desde infecçóes até lesóes resultantes de mau posicionamento cirúrgico, além de queimaduras, de funcionamento inadequado de aparelhos e, até mesmo, havendo realização de cirurgia em local errado [9].

Dentre os 14 artigos analisados, seis tratam do papel da enfermagem na promoção da segurança do paciente. Desses, quatro abordam o paciente hospitalizado e dois focam no paciente cirúrgico.

A enfermagem é responsável por atividades que variam desde a provisáo e a previsão de materiais até o contato constante com setores de manutenção, laboratório, compras, entre outros. As responsabilidades do enfermeiro para garantir um procedimento cirúrgico seguro tornam-se mais complexas à medida que é necessário integrar competências técnica, assistencial, de pesquisa e administrativa. Além disso, também é importante que o enfermeiro tenha uma equipe preparada e qualificada para solucionar possíveis intercorrências no período perioperatório e, principalmente, para agir com segurança em todos os procedimentos que apoiam a cirurgia [10].

Como o enfermeiro tem papel importante na promoção da segurança do paciente durante o processo assistencial, não sendo possível evitar ou prevenir eventos adversos, deve dar assistência às necessidades do paciente, por ter as informaçóes referentes à pertinência e à atividade do paciente na unidade, além de ser referência aos demais profissionais para informar eventos adversos, incidentes, ocorrências administrativas e fazer solicitaçóes [12].

A enfermagem deve estar ciente de que a complexidade do desafio e do seu discurso depende de sua disposição em assumir a liderança de segurança, tendo como elementos imprescindíveis a pesquisa, a formação e a ideia de que a segurança do paciente é a segurança de todos. Por isso, os pacientes identificam os profissionais de enfermagem como seus defensores, já que lhes prestam cuidados ininterruptos [14].

Além disso, a enfermagem também mostra algumas dificuldades em relaçáo à segurança do paciente. Um estudo aponta a percepção dos enfermeiros entrevistados. Nele, 3,62\% responderam ser difícil falar abertamente quando percebem algum problema com o cuidado do paciente. Já $49 \%$ dos profissionais afirmaram que a cultura em seu local de trabalho náo é apropriada para aprender com o erro dos outros. Do total, $82 \%$ concordaram que, quando a carga de trabalho é excessiva, o desempenho torna-se prejudicado, e isso, consequentemente, prejudica a segurança do paciente. Outro fator que influencia é a insatisfação do profissional pelo trabalho, devido ao acúmulo de atividades e as escassas perspectivas de obter novos conhecimentos, o que prejudica a qualidade do seu desempenho junto ao paciente. Apesar disto, a maioria dos profissionais que participou do referido estudo diz estar satisfeita com o seu trabalho e se sentiria segura se fosse tratada como paciente no setor em que trabalha. Entretanto, eles admitem não conhecer os meios adequados para encaminhar as questóes relacionadas à segurança do paciente [15].

A enfermagem atua em diversos campos, sendo alguns táo desgastantes que podem levar a episódios de estresse. As tarefas com elevado grau de responsabilidade e exigência podem criar tensóes para o profissional, para a equipe e para a comunidade, podendo colocar em risco a segurança do paciente. $\mathrm{O}$ medo e o nervosismo também podem acarretar erros que comprometem o bem-estar do paciente. Além disso, o profissional pode perceber o seu ambiente de trabalho como ameaçador às suas necessidades de realização pessoal e profissional, fato que prejudica a interação com suas funções e com o local de trabalho. Esse fato também ocorre muito comumente com acadêmicos de enfermagem, que 
passam por bastante estresse, medo e nervosismo, pois estáo aprendendo e, ao mesmo tempo, sendo avaliados pelos professores. A tensão a que os acadêmicos são submetidos pode contribuir para o desgaste psicológico e também para a diminuição de concentração e falhas da memória, levando ao aparecimento de eventos adversos e práticas não seguras. Sendo assim, é possível perceber que o estresse é um fator que influencia não só aqueles que estão cursando enfermagem, mas também os que já são profissionais, podendo gerar graves influencias no cuidado do paciente. Vale lembrar que o cuidado de enfermagem ocorre geralmente em momentos de fragilidade humana, fato que contribui para que haja a exposiçáo tanto de quem cuida, quanto de quem recebe os cuidados a uma variedade de emoçóes intrínsecas a esse cuidado [16]

Mesmo com o esforço dos profissionais de saúde em proporcionar uma melhor assistência aos pacientes, isso náo os impede de cometer falhas e provocar acidentes decorrentes do cuidado prestado. Com base nisso, a fragmentação assistencial exige um novo modelo que favoreça a comunicação e o trabalho em equipe, dado o aumento da complexidade dos procedimentos, o uso de tecnologias e a expansão do conhecimento médico, a fim de proporcionar a melhor segurança ao paciente [17].

Um dos fatores que dificultam o desenvolvimento de açóes a favor da segurança é a grande pressão assistencial que sofrem os profissionais de saúde. Então, a grande demanda assistencial e a intensa carga de trabalho tendem a impedir a procura de evidências científicas. Outro fator preocupante nesse processo é a desconfiança do paciente em relaçáo aos profissionais e vice-versa, o que leva à perda de credibilidade e à falta da participaçáo dos pacientes nas decisóes clínicas. Assim, os profissionais têm a sensação de que a segurança é algo real e de qualidade, mas que, com o passar do tempo, será esquecida. Isso denota a falta de responsabilidade e a resistência à mudança [14].

Torna-se relevante, portanto, mostrar aos profissionais o quanto suas atitudes podem influenciar nos cuidados com o paciente. Isso pode ser feito em grupos com a equipe de trabalho, com uma comunicação clara, havendo oportunidade para todos exporem suas dificuldades e, juntos, construírem um cuidado mais eficiente.

Outro fator importante na construção de um trabalho eficiente é a cultura de náo punição. Muitos profissionais escondem os erros cometidos, pois têm medo de serem punidos. Portanto, eles precisam ser incentivados a notificarem tais erros para que sejam feitas medidas de prevenção. Muitas das razóes que levam o profissional a não relatar os incidentes são sentimentos como vergonha, autopuniçáo, medo da crítica de outras pessoas e do litígio. Para que este relato seja eficaz é preciso um esforço para mostrar aos profissionais que seu objetivo é melhorar a segurança e nunca acusá-los ou puni-los. Portanto, é necessário trocar a cultura punitiva por uma cultura de monitorização contínua dos riscos reais e potenciais [10].

É importante que a instituição de saúde estimule essa cultura de não punição, incentivando a notificação dos eventos adversos e a implementaçáo de açóes que previnam sua ocorrência [4]. Oliveira, Caregnato e Hoefel [9] incentivam a criação de medidas para análise crítica das situaçóes de risco e enfatizam a necessidade das notificaçóes dos EAs. Eles ainda referem que muitas instituiçóes usam a cultura da culpa e punição, e isso, além de desestimular a notificação dos EAs, revigora a omissão dos trabalhadores frente as tais situaçóes. Fica, pois, evidente que é preciso reforçar a disseminação do conhecimento e o aprendizado para minimizar erros. Muitos profissionais, por medo de serem recriminados, muitas vezes não notificam os EAs ocorridos. No entanto, o registro deste tipo de ocorrência é importante, já que auxilia a criação de medidas preventivas.

Ressalte-se que essas notificaçóes são feitas por boletins de notificação de eventos adversos (BNEA), instrumentos de comunicação entre os profissionais de enfermagem e a diretoria da instituição sobre os EAs. Nesse boletim constam os seguintes campos: identificaçáo do paciente e da unidade, tipos de ocorrências, espaço destinado ao relato da ocorrência e espaço destinado à descrição da conduta do notificador frente ao fato. As ocorrências são separadas em dois tipos: EA/incidente e ocorrência administrativa (ocorrência que náo envolveu diretamente o paciente). A realização destas notificações torna-se um mecanismo que garante a prevençáo e minimização dos erros, visto que se devem analisar as causas desses erros para, entáo, serem estabelecidas açóes corretivas, informando-se os profissionais sobre o desenvolvimento da análise [12].

$\mathrm{O}$ ato de notificar os erros cometidos deve ser estimulado na equipe, uma vez que sua análise repercute na prevenção de EAs, não podendo mais ser considerado como motivo de reprimir o profissional, 
mas sim subsidiar o planejamento de estratégia que minimizem sua ocorrência e reconheça o erro como parte integrante do sistema [3].

A enfermagem e seus profissionais compóe a última barreira de proteção e defesa do doente contra a ocorrência de EAs. Daí decorre a importância de preparar esses profissionais para aprenderem a identificar, prevenir e notificar os EAs [4]. Os eventos adversos, sempre que são identificados, precisam ser analisados e investigados detalhadamente. Uma maneira de fazer esse trabalho é por meio da abordagem baseada em sistemas que encaixam todas as atividades na organização, contribuindo para a manutenção e a melhoria da segurança do paciente. A prevenção desses eventos é pré-requisito dessa segurança. Uma política de tolerância-zero é o único padrão que pode ser aceito pelos pacientes e pelo público em geral, e pode eticamente ser justificado por instituiçóes de saúde. A implantação dessa política pode enfrentar barreiras culturais e organizacionais, principalmente pela equipe do centro cirúrgico. Uma das grandes barreiras enfrentadas é a falta de treinamento da equipe, o náo comprometimento da instituição e a não adesão dos profissionais a protocolos [7].

No que se refere a protocolos, o mais usado é o Protocolo Universal, utilizado por instituiçóes governamentais e privadas para a prevençáo de eventos relacionados a este tipo de procedimentos. A frequência de erros é considerada um dos indicadores de qualidade da assistência, sendo um dos pontos avaliados em alguns processos de acreditação. Dessa forma, para garantir a qualidade da assistência ao paciente cirúrgico, foi criado, em 2003 pela Join Commission Board of Comissioners, o Protocolo Universal para "Prevenção do lado errado, procedimento errado e paciente errado" [7].

Esse protocolo tem a finalidade de eliminar situaçóes desastrosas, decorrentes de cirurgias ou de procedimentos invasivos por meio de três etapas fundamentais: a) identificação da pessoa operada, da cirurgia ou do procedimento e do local de intervenção, em três momentos [de agendado, na admissão e antes do ingresso à sala de operação]; b) marcação do local cirúrgico em todos os casos que envolvam lateralidade, após o preparo do paciente; c) verificaçáo, envolvendo toda a equipe, do sítio operatório antes do início do procedimento [17].

Vale lembrar que a enfermagem tem um importante papel na utilização deste protocolo, uma vez que os enfermeiros devem verificar que o pro- cedimento cirúrgico não comece antes que todas as etapas sejam cumpridas. Por isso, esses profissionais devem ser leais e comprometidos com a segurança do paciente em interação com a equipe cirúrgica a fim de garantir que ocorra uma verificação final. Isso pode ser feito pela utilização de um check list com todas essas informaçóes necessárias para realizar o procedimento cirúrgico com segurança [7].

\section{Conclusão}

A partir da constatação de que a enfermagem tem um papel fundamental na prevenção e promoção da segurança do paciente, é importante ressaltar que uma das açóes de enfermagem que visa a esse objetivo é o enfermeiro preparar e qualificar sua equipe para que essa saiba atuar em momentos difíceis que possam causar danos ao paciente. Além disso, a cultura de não punição também é muito importante para prevenir eventos adversos, pois ela mostra aos profissionais que quando se cometem erros náo se deve ter medo e vergonha do que aconteceu, e sim notificar esse ato para que, a partir dele, possam buscar estratégias para evitar a recorrência de erros. Sendo assim, as instituiçóes de saúde devem se preocupar com esse tema e abordá-lo com seus funcionários, divulgando conceitos claros sobre os tipos de eventos adversos.

\section{Referências}

1. Grigoleto ARL, Gimenes FRE, Avelar MCQ. Segurança do cliente e as açóes frente ao procedimento cirúrgico. Rev Eletrônica Enferm 2011;13(3):347-54.

2. Conselho Regional de Enfermagem do Estado de São Paulo - COREN. 10 passos para a segurança do paciente. [internet]. 2010. [citado 2013 Out 10]. Disponível em URL: http://inter.coren-sp.gov.br

3. Carneiro FS, Bezarr ALQ, Silva AEBC, Paranaguá TTB, Branquinho, NCSS. Eventos adversos na clínica cirúrgica de um hospital universitário: instrumento de avaliação da qualidade. Rev Enferm UERJ 2011;19(2):204-11.

4. Silva RCL, Cunha JJSA, Moreira CLS. Evento adverso em terapia intensiva: o que sabem os profissionais de enfermagem. Rev Pesqui Cuid Fundam 2011;3(2):1848-55.

5. Organização Mundial da Saúde; Organização Pan-Americana da Saúde; Brasil, Ministério da Saúde, Agência Nacional de Vigilância Sanitária. Segundo desafio global para a segurança do paciente: cirurgias seguras salvam vidas: orientaçóes para cirurgia segura da OMS. Rio de Janeiro: OMS; 2009.

6. Pedreira MLG. Práticas de enfermagem baseadas em evidências para promover a segurança do paciente. Acta Paul Enferm 2009;22(Especial - 70 Anos):880-1. 
7. Vendramini RCR, Silva EA, Ferreira KASL, Possari JF, Baia WRM. Segurança do paciente em cirurgia oncológica: experiência do Instituto do câncer do Estado de São Paulo. Rev Esc Enferm USP 2010;44(3):827-32.

8. Nascimento NB, Travassos CMR. O erro médico e a violação às normas e prescriçóes em saúde: uma discussão teórica na área de segurança do paciente. Physis 2010;20(2):625-51.

9. Oliveira BC, Caregnato RCA, Hoefel HHK. Gerenciamento de riscos: qualidade na assistência perioperatória. Rev SOBECC 2010;15(4):21-7.

10. Souza LP, Bezarra ALQ, Silva AEBC, Carneiro FS, Paranaguá TTB, Lemos LF. Eventos adversos: instrumento de avaliação do desempenho em centro cirúrgico de um hospital universitário. Rev Enferm UERJ 2011;19(1):127-33.

11. Moura MLO, Mendes W. Avaliação de eventos adversos cirúrgicos em hospitais do Rio de Janeiro. Rev Bras Epidemiol 2012;15(3):523-35.

12. Paiva MCMS, Paiva SAR, Berti HW. Eventos adversos: análise de um instrumento de notificação utilizado no gerenciamento de enfermagem. Rev Esc Enferm USP 2010;44(2):287-94.

13. Mendes W, Travassos C, Martins M, Noronha JC. Revisão dos estudos de avaliação da ocorrência de eventos adversos em hospitais. Rev Bras Epidemiol 2005;8(4):393-406.

14. Ques AA, Montoro CH, Gonzáliz MG. Fortalezas e ameaças em torno da segurança do paciente segundo a opinião dos profissionais de enfermagem. Rev Latinoam Enferm 2010;18(3):42-9.

15. Rigobello MCG, Carvalho REFL, Cassiani SHB, Galon T, Capucho HC, Deus NN. Clima de segurança do paciente: percepçáo dos profissionais de enfermagem. Acta Paul Enferm 2012;25(5):728-35.

16. Santos VEP, Radünz V. O estresse das acadêmicas de enfermagem e a segurança do paciente. Rev Enferm UERJ 2011;19(4):616-20.

17. Cunha ALM, Acunã AA, Bispo DM. Estratégias de prevenção de eventos adversos na sala operatória. Rev SOBECC 2007;12(3):20-3. 